\title{
B-06
}

\section{INFLUENCIA DE LA FERTIRRIGACIÓN CON AGUA REGENERADA Y LAS LABORES DE MANTENIMIENTO EN LA UNIFORMIDAD DE RIEGO}

Contreras París, J.I.(1), López Segura, J.G. (1), Trujillo Delgado, J. (2), Alonso López, F.(1) , Baeza Cano, R. (1)

\author{
${ }^{1}$ Instituto de Investigación y Formación Agraria y Pesquera de Andalucía (IFAPA), Centro La \\ Mojonera, Camino San Nicolás, n¹, 04745 La Mojonera, Almería. \\ juanai.contreras@juntadeandalucia.es \\ ${ }^{2}$ Universidad de Almería, Ctra. De Sacramento s/n, 04120 Almería.
}

\section{Resumen}

El objetivo del trabajo ha sido estudiar el efecto de la fertirrigación y las labores de mantenimiento en la uniformidad de distribución de caudal utilizando agua urbana regenerada. El ensayo se desarrolló en un invernadero experimental en el Centro IFAPA La Mojonera en La Cañada (Almería). Se estableció un diseño experimental factorial con dos factores, manejo del riego y tipo de emisor. En lo que respecta al factor manejo de riego se dispusieron tres tratamientos, AR-Riego con agua residual urbana regenerada; ARFRFertirriego con agua residual urbana regenerada+equilibrio fertilizante estándar y ARFRMFertirriego con agua residual urbana regenerada+equilibrio fertilizante estándar +mantenimiento. En el factor tipo de emisor, se evaluaron 20 modelos comerciales de goteros seleccionados en estudios previos de diferentes tipologías. Se determinó el coeficiente de uniformidad de caudal y el porcentaje de obturación a las 0, 40, 60, 80 y $100 \mathrm{~h}$ de funcionamiento. De los resultados obtenidos se puede concluir que el tratamiento de riego afectó al porcentaje de obturación, siendo mayor en los tratamientos fertirrigados. Las labores de mantenimiento predefinidas mejoraron la uniformidad pero no resultaron eficaces para evitar la obturación, por lo que sería necesario redefinir las labores de mantenimiento más adecuadas a este tipo de agua.

\section{1- Introducción y Objetivos}

La horticultura intensiva desarrollada en el Sureste de España ha sufrido, durante sus más de 50 años de trayectoria, una serie de adaptaciones y cambios propiciados por la incorporación de nuevas tecnologías y como respuesta a los problemas que han ido surgiendo. En lo que respecta a los recursos hídricos, se ha producido un descenso, en términos de calidad y cantidad, de las reservas de agua subterránea (CMAOT, 2012), fuente principal de abastecimiento del sistema productivo. Por este motivo, se ha hecho necesario la incorporación de nuevas fuentes de agua como las Residuales Urbanas Regeneradas (ARUR). Este tipo de agua, en general se caracterizan por presentar una buena calidad química, sin embargo una peor calidad física y biológica, en comparación con las tradicionales aguas subterráneas. Como consecuencia del empleo de estas aguas, en las instalaciones de riego localizado, se han detectado problemas relacionados con el mantenimiento y mayores niveles de obturación de los emisores de riego (Baeza et al. 2010).

Los principales agentes causantes de obturación no son exclusivamente los sólidos en suspensión, sino la aglomeración de estos (partículas finas minerales u orgánicas) con 
subproductos microbianos y biomasa desarrollada en el interior de las tuberías y ramales. Este problema se agrava por la incorporación de nutrientes en el agua de riego, fertirrigación, ya que estos nutrientes sirven de alimento a los microorganismos presentes en el agua, incrementando la biomasa presente.

Estudios previos, realizados por el IFAPA, demuestran que las instalaciones de riego en las fincas que utilizan estas aguas presentan habitualmente problemas de funcionamiento y uniformidad relacionados con frecuencia a obturaciones en los emisores (Baeza et al., 2010). Además, estudios complementarios concluyen que estos problemas pueden ser de mayor o menor magnitud dependiendo del tipo de emisor instalado (Baeza et al., 2012; Contreras et al., 2013).

El objetivo del trabajo fue estudiar el efecto de la fertirrigación y las labores de mantenimiento en la uniformidad de distribución de caudal utilizando agua urbana regenerada.

\section{2- Materiales y Métodos}

El ensayo se desarrolló en un invernadero experimental multitúnel de $1200 \mathrm{~m}^{2}$, situado en el Centro IFAPA La Mojonera en La Cañada (Almería) (Fotografía 1) y equipado con control automático de riego. Este invernadero disponía de un banco de pruebas de emisores, preparado para evaluar 240 líneas de riego. Se estableció un diseño experimental factorial con dos factores, manejo del riego y tipo de emisor, en bloques al azar con tres repeticiones. La unidad experimental básica fue un lateral de riego.

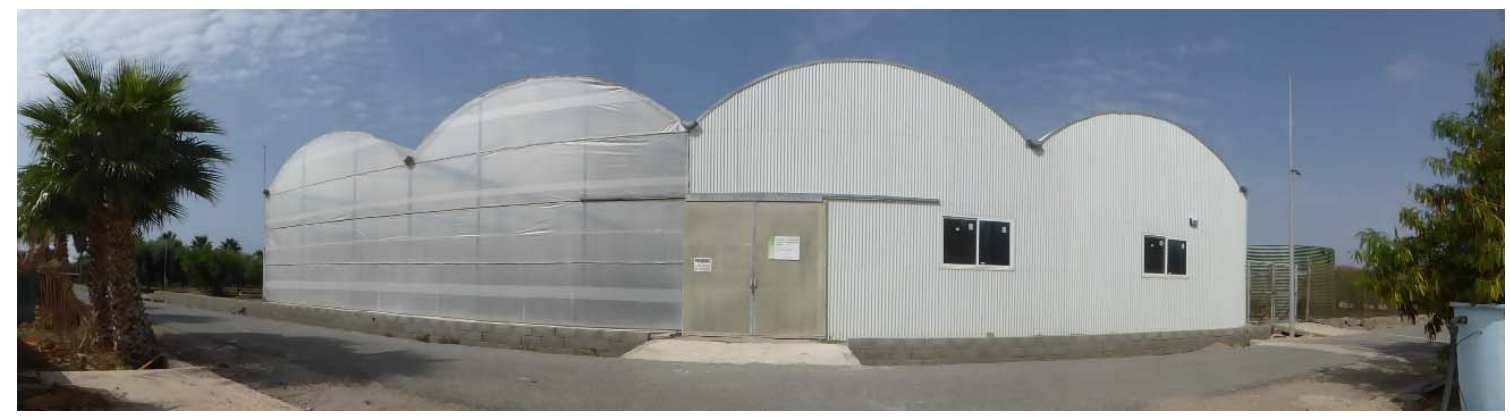

Fotografía 1. Frontal del invernadero multitúnel usado en la realización del ensayo.

En lo que respecta al factor manejo de riego se dispusieron tres tratamientos:

1. AR - Riego con agua residual urbana regenerada. estándar.

2. ARFR - Fertirriego con agua residual urbana regenerada+equilibrio fertilizante

3. ARFRM - Fertirriego con agua residual urbana regenerada+equilibrio fertilizante estándar+mantenimiento.

A los tratamientos AR y ARFR no se les realizó labor alguna de mantenimiento, en cambio en el ARFRM se realizó:

-Apertura durante varios segundos de los tapones finales de los ramales de riego para la limpieza de los sedimentos. Esta labor se realizaba con una periodicidad mensual.

-Mantenimiento del pH de la solución de riego en valores constantes de pH 6 para evitar la formación de precipitados. 
En el factor tipo de emisor, se evaluaron 20 modelos comerciales de goteros seleccionados en estudios previos de diferentes tipologías, y que se recogen en la Tabla 1.

Tabla 1. Emisores analizados.

\begin{tabular}{|c|c|c|c|c|}
\hline & Modelo & Fabricante & $\begin{array}{l}\text { Tipo de } \\
\text { Inserción }\end{array}$ & $\begin{array}{l}\text { Compensación } \\
\text { de Caudal }\end{array}$ \\
\hline 1 & $\begin{array}{l}\text { T D Advanced } \\
\text { OL }\end{array}$ & Mondragon & Pinchado & NO \\
\hline 2 & ADO & Caudal & Pinchado & SI \\
\hline 3 & Interlínea 12 & Hidroten & Interlínea & NO \\
\hline 4 & NYA & Primaram & Interlínea & NO \\
\hline 5 & PCJ & Netafim & Pinchado & SI \\
\hline 6 & $\begin{array}{l}\text { Cónico } \\
\text { desmontable }\end{array}$ & Hidroten & Pinchado & NO \\
\hline 7 & PC-CNL & Netafim & Pinchado & SI \\
\hline 8 & TD Advanced M & Mondragon & Pinchado & NO \\
\hline 9 & Hydrogol & John DeereWater & Integrado & NO \\
\hline 10 & AGR & Azud & Interlínea & NO \\
\hline 11 & AmnonDrip & NaanDanJainlbérica & Integrado & SI \\
\hline 12 & Interlínea 16 & Hidroten & Interlínea & NO \\
\hline 13 & Interlínea 16 & Amacom & Interlínea & NO \\
\hline 14 & PRO & Azud & Integrado & NO \\
\hline 15 & Megadrip & Mondragon & Integrado & SI \\
\hline 16 & Topdrip & NaanDanJainlbérica & Integrado & NO \\
\hline 17 & Hydro PC & John DeereWater & Integrado & SI \\
\hline 18 & UniRam & Netafim & Integrado & SI \\
\hline 19 & LIN & Extruline Caudal & Integrado & NO \\
\hline 20 & ARIES & Netafim & Integrado & NO \\
\hline
\end{tabular}

Se realizaron pulsos continuos de riego de 30 minutos, en 5 aplicaciones semanales durante un periodo de 37 semanas, equivalente a una campaña de riego en un ciclo medio de cultivos hortícolas en invernadero. El agua utilizada fue agua residual urbana procedente de la ciudad de Almería y regenerada mediante tratamiento terciario de filtración, ozonización y/o cloración. Los parámetros del agua utilizada se muestran en la Tabla 2. 
Tabla 2. Parámetros medios presentes en el agua de riego e intervalo de variación. $\mathbf{N}$ (número de muestras)=13.

\begin{tabular}{|c|c|c|c|}
\hline Parámetros & Media & Mínimo & Máximo \\
\hline Sólidos en suspensión $(\mathrm{mg} / \mathrm{l})$ & 16,93 & $<2$ & 156 \\
\hline E.coli (u.f.c./100ml) & 0 & 0 & 0 \\
\hline $\mathrm{pH}$ & 7,47 & 7,2 & 7,8 \\
\hline Turbidez U.N.T. & 8,4 & 1,3 & 64 \\
\hline Cloro libre residual mg Cl $/ / \mathrm{l}$ & 1,62 & 0,12 & 4,72 \\
\hline Salmonella spp (en un litro) & \multicolumn{3}{|c|}{ Ausencia } \\
\hline Huevos de nematodos intestinales & 4,07 & 0 & 53 \\
\hline $\mathrm{DBO}_{5}\left(\mathrm{mgO}_{2} / \mathrm{l}\right)$ & 5 & $<5$ & 34 \\
\hline $\mathrm{DQO}_{\left(\mathrm{mgO}_{2} / \mathrm{l}\right)}$ & 52,53 & 24 & 246 \\
\hline
\end{tabular}

Se determinó el coeficiente de uniformidad de caudal y el porcentaje de obturación a las $0,40,60,80$ y $100 \mathrm{~h}$ de funcionamiento.

La uniformidad se determinó por el método clásico del cuarto inferior propuesto por Merriam y Keller (1978), y cuya fórmula se recoge en la Ecuación 1.

$$
\text { CUC }=\frac{9_{\text {mat }}}{9_{m}} \cdot 100 \quad(\text { Ecuación } 1)
$$

Siendo:

CUC el Coeficiente de Uniformidad de Caudal, en tanto por 100.

$\mathbf{q}_{25 \%}$ el promedio de los caudales del $25 \%$ de los emisores, cuyo caudal sea de menor valor.

$\mathbf{q}_{\mathbf{m}}$ el promedio del caudal del total de los emisores.

Según el Coeficiente de Uniformidad de Caudal (CUC) obtenido, la calificación de la instalación se realizará según se muestra en la Tabla 3.

Tabla 3. Calificación de la instalación de riego, en función del Coeficiente de Uniformidad de Caudal (CUC) (Merriam y Keller, 1978).

\begin{tabular}{|c|c|}
\hline CUC & Calificación \\
\hline$>95 \%$ & Excelente \\
\hline $85 \%-95 \%$ & Buena \\
\hline $80 \%-85 \%$ & Aceptable \\
\hline $70 \%-80 \%$ & Pobre \\
\hline$<70 \%$ & Inaceptable \\
\hline
\end{tabular}


El porcentaje de obturación medio se ha determinado como la reducción de caudal presentada por los emisores, según la Ecuación 2.

$$
Q_{\text {nobturectôn }}=\frac{Q_{\mathrm{Q}}-\varphi_{m}}{Q_{\mathrm{Q}}} \cdot 100 \quad \text { (Ecuación 2) }
$$

Siendo:

$\mathbf{Q}_{0}$ el Caudal obtenido en el $1^{\mathrm{er}}$ muestreo.

$\mathbf{Q}_{\mathbf{n}}$ el Caudal obtenido en el muestreo $\mathrm{n}$.

Los resultados fueron analizados estadísticamente con el software STATGRAPHICS Plus (versión 5.1). Se realizó un análisis multifactorial ANOVA para identificar la significación de los factores estudiados. Cuando el análisis estadístico reveló diferencias significativas entre tratamientos se aplicó un test de comparación de media (LSD; mínima diferencia significativa) con $\mathrm{p} \leq 0.05$.

\section{3- Resultados y conclusiones}

El efecto de los factores estudiados y la interacción entre ambos en la uniformidad de caudal se muestra en la Tabla 3. El tratamiento de riego solo tuvo efecto al final del ensayo, a las $100 \mathrm{~h}$ de trabajo, por lo que es un efecto que se produce a largo plazo. Sin embargo, el modelo de emisor presentó influencia desde el inicio del ensayo hasta el final $(0,40,60,80$ y 100 horas de trabajo). No existió interacción entre el modelo de emisor y los distintos tratamientos.

Tabla 3. Análisis factorial de la varianza, nivel de significación e interacción de los factores

CUC (\%)

\begin{tabular}{cccccc}
\hline Factor & $\mathbf{0}$ horas & $\mathbf{4 0}$ horas & $\mathbf{6 0}$ horas & $\mathbf{8 0}$ horas & $\mathbf{1 0 0}$ horas \\
\hline A: Tratamiento riego & $\mathrm{ns}$ & $\mathrm{ns}$ & $\mathrm{ns}$ & $\mathrm{ns}$ & $*$ \\
B: Modelo de emisor & $* \star$ & $* \star$ & $*$ & $*$ & $*$ \\
Interacción AxB & $\mathrm{ns}$ & $\mathrm{ns}$ & $\mathrm{ns}$ & $\mathrm{ns}$ & $\mathrm{ns}$ \\
\hline
\end{tabular}

ns, no significativo. * *Significativo para $p<0.05 . \quad$ **Significativo para $p<0.01$

En la Figura 1 se muestran los valores medios del CUC (\%) a las 100 horas de trabajo, para los diferentes tratamientos de riego. El tratamiento fertirrigado con labores de mantenimiento (ARFRM), con independencia del modelo de emisor, presentó al finalizar en ensayo, un coeficiente de uniformidad de caudal significativamente mayor que el tratamiento sin labores de mantenimiento (ARFR). El tratamiento ARFR mostró un CUC de 92,7\% que se diferenció estadísticamente de los tratamientos AR (95,0\%) y ARFRM (94,8\%).

La mejora de la uniformidad en el tratamiento de fertirrigación más mantenimiento frente al tratamiento de fertirrigación sin mantenimiento pudo deberse a que las labores realizadas de mantenimiento hayan evitado precipitados y acumulación de los mismos en los tramos finales de las tuberías. 
Media e intervalo LSD al 95\%

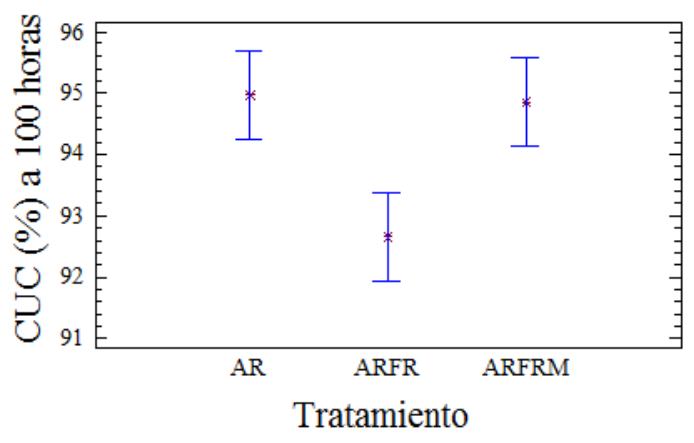

Figura 1. Valores medios e intervalo de confianza $(p<0,05)$ del coeficiente de Uniformidad de Caudal (\%) a las 100 horas de trabajo (CUC), para los diferentes tratamientos de riego.

La Figura 2 muestra los valores medios del CUC (\%) a las 0, 40, 6080 y 100 horas de riego, del total de 20 modelos de emisores de riego analizados. Todos los modelos de emisores analizados al comienzo de su ciclo de trabajo (0 horas) presentaron una alta uniformidad independientemente del tratamiento, sin embargo, al final del ciclo de trabajo (100 horas) la mayor parte de modelos de emisores redujeron ligeramente el CUC.

Los CUC obtenidos por los modelos de emisores evaluados fueron elevados, clasificándose como excelentes o buenos en todas las mediciones. A las 0 horas de riego el $85 \%$ de los modelos de emisores se clasificaron como excelente, conservándose esta distribución tras 40 horas de riego. A las 60 horas de riego el $80 \%$ de los emisores se clasificaban como excelente descendiendo al $50 \%$ a las 80 horas. Al finalizar el ensayo (100 horas de riego) solo el $40 \%$ los modelos de emisores que se clasificaron como excelente.

En cuanto a la reducción de caudal tras las 100 horas de riego, tanto el tratamiento como el modelo de emisor mostraron efecto, sin presentar interacción entre ellos (Tabla 4). 
Media e intervalo LSD al 95\%

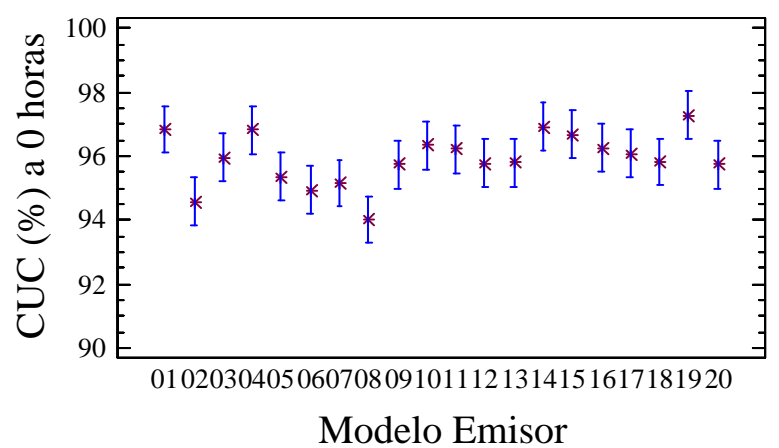

Media e intervalo LSD al 95\%

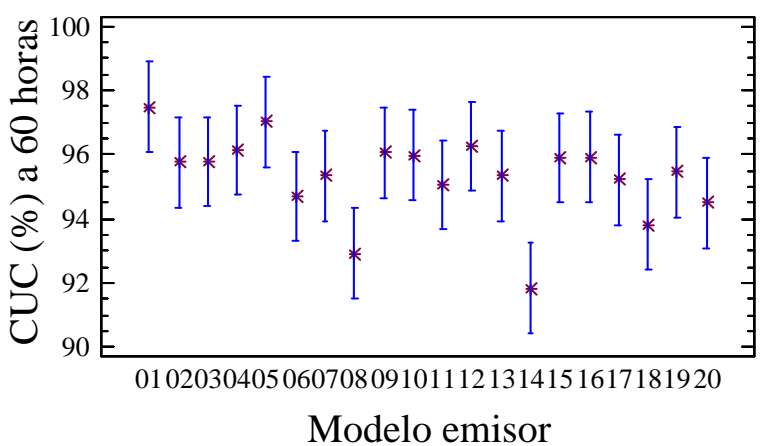

Modelo emisor
Media e intervalo LSD al 95\%

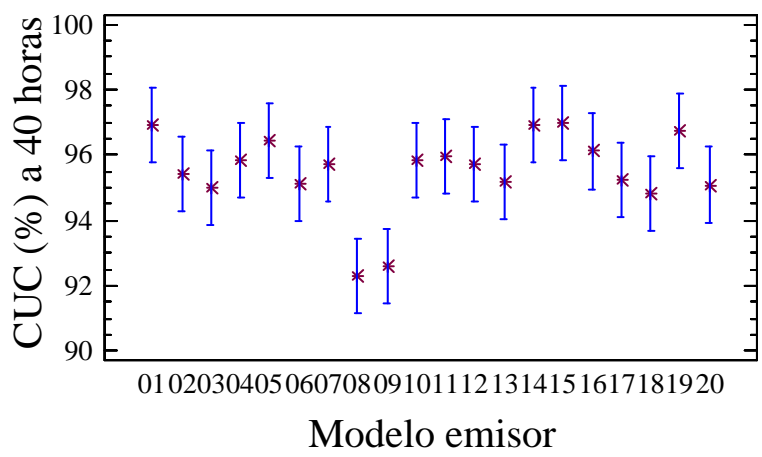

Media e intervalo LSD al 95\%

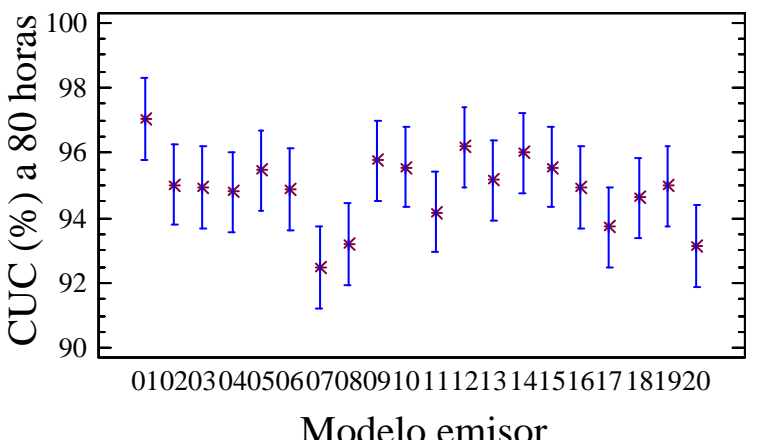

Media e intervalo LSD al 95\%

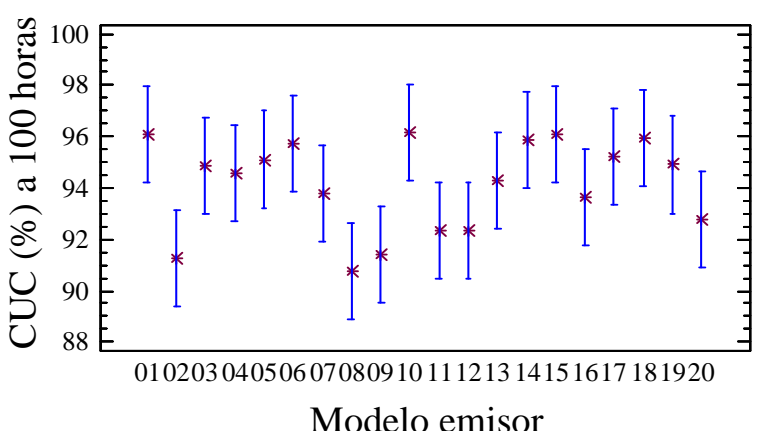

Figura 2. Valores medios e intervalo de confianza $(p<0,05)$ del coeficiente de Uniformidad de Caudal (\%) a las 0, 40, 6080 y 100 horas de trabajo (CUC), de los 20 modelos analizados.

Tabla 4.Nivel de significación e interacción de los factores en cuanto a la reducción del caudal.

Reducción de caudal (\%)

\begin{tabular}{cc}
\hline Factor & Nivel de significación \\
\hline A: Tratamiento & $*$ \\
B: Modelo de emisor & $* *$ \\
Interacción AxB & ns \\
\hline
\end{tabular}

$n s$, no significativo. $\quad$ *Significativo para $p<0,05 . \quad$ **Significativo para $p<0,01$ 
Independientemente del modelo del emisor, tras 100 horas de riego, los tratamientos con fertirrigación mostraron una reducción de caudal significativamente mayor que el tratamiento sin fertirrigación (Figura 3). La reducción de caudal se cifró en un 4,0 y 4,6\% para los tratamientos ARFR y ARFRM, respectivamente, sin embargo el tratamiento AR solo mostró una reducción del 1,3\%, mostrando la influencia que tiene la fertirrigación sobre la obturación de los emisores.

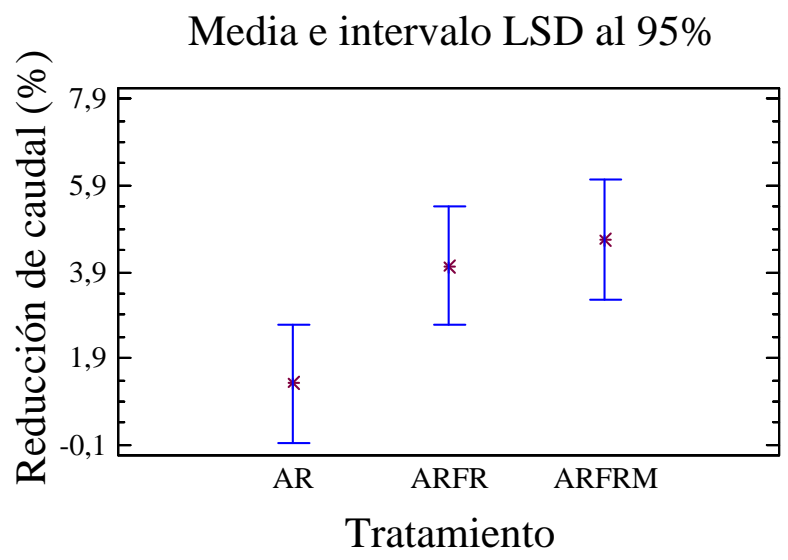

Figura 3. Reducción de caudal (\%) tras 100 horas de trabajo de los tratamientos estudiados, con la representación del intervalo de Mínima Diferencia Significativa (LSD) al 95\%.

En lo que respecta al modelo de emisor, en la mayoría se produjo una reducción del caudal (Figura 4), cabe destacar el modelo 6 con una reducción del 16\%. Se pudo observar que en algunos modelos de emisores, no se produjo una reducción del caudal, sino que se produjo un incremento, esto pudo ser debido a que estos emisores eran compensantes y el aumento de caudal pudo estar asociado a una afección al mecanismo de membrana.

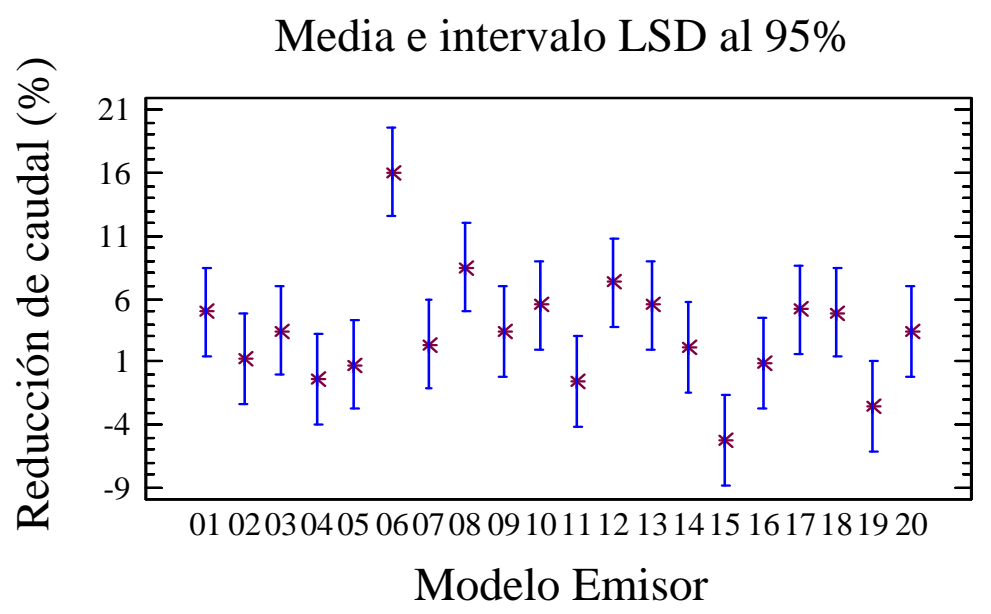

Figura 4. Reducción de caudal (\%) tras 100 horas de trabajo de los 20 modelos analizados, con la representación del intervalo de Mínima Diferencia Significativa (LSD) al 95\%.

De los resultados obtenidos se puede concluir que el tratamiento de riego afecta al porcentaje de obturación, siendo mayor en los tratamientos fertirrigados. Las labores de mantenimiento predefinidas mejoraron la uniformidad pero no resultaron eficaces para evitar la obturación, por lo que sería necesario redefinir las labores de mantenimiento más adecuadas a este tipo de agua. 


\section{4- Bibliografía}

Baeza Cano, R., Contreras París, J.I., Martín Usero, F., Zapata Sierra, A. \& López Segura J.G. (2012). Estudio prospectivo de los emisores de riego localizado instalados en la zona regable del Bajo Andarax con aguas residuales urbanas regeneradas. XXX Congreso Nacional de Riegos. Albacete, 12-14 de junio de 2012.

Baeza Cano, R., Gavilán Zafra, P., Del Castillo Lupiáñez, N., Berenguel, P. \& López Segura, J.G. (2010). Programa de evaluación y asesoramiento en instalaciones de riego en invernadero con uso de dos fuentes distintas de agua: subterránea y regenerada. XXXVIII Congreso Nacional de Riegos. León 15-17 Junio de 2010.

Consejería de Medio Ambiente y Ordenación del Territorio (CMAOT). (2012). Demarcación Hidrográfica de las Cuencas Mediterráneas Andaluzas. Documentos plan hidrológico de la demarcación hidrográfica de las cuencas mediterráneas andaluzas. Consejería de Medio Ambiente y Ordenación del Territorio. Junta de Andalucía.

Contreras París, J.I., Baeza Cano, R, López Segura J.G. \& Gavilán Zafra, P. (2013). Comportamiento de emisores de riego localizado de bajo caudal con aguas residuales urbanas regeneradas. Estudio prospectivo de los emisores de riego localizado instalados en la zona regable del Bajo Andarax con aguas residuales urbanas regeneradas. XXXI Congreso Nacional de Riegos. Orihuela, Alicante, 18-20 de junio de 2013.

Merrian, J.L \& Keller, J. (1978). Farm irrigation system evaluation: a guide for management. Utah State University. 